\title{
The role of the urban community hospital in gonorrhoea surveillance
}

\author{
R. C. RENDTORFF, J. W. CURRAN, R. W. CHANDLER, S. GLASSCO, \\ and L. WISER \\ From the Departments of Community Medicine, Medicine, Microbiology, and Obstetrics and Gynecology, \\ University of Tennessee Centrer for the Health Sciences, and Memphis-Shelby County Health Department, USA
}

\section{Summary}

In a progressive surveillance programme designed to detect gonococcal infection in females, 113,063 women were screened for gonorrhoea in MemphisShelby County, Tennessee, during a 2-year period.

The importance of including the urban community hospital in the surveillance programme is emphasized by the contribution of the City of Memphis Hospital where 53.3 per cent. of all positive cases of gonorrhoea were detected in only 34.2 per cent. of all tests done. The City of Memphis Hospital emergency room was an especially productive surveillance area, giving 29.0 per cent. of all positives with only $6 \cdot 1$ per cent. of all tests performed.

The importance of taking routine cultures for gonococci from women presenting for prenatal care, delivery, or for genitourinary complaints in an urban hospital is stressed.

\section{Introduction}

The current epidemic of gonorrhoea in the United States has stimulated increased expenditure in the control of this disease. In an effort to identify asymptomatic or otherwise unrecognized cases in women, a portion of these funds has been directed to surveillance efforts through culture screening. Adequate treatment and follow-up of these cases should serve to contain the spread of gonorrhoea by reducing the female reservoir of infection.

Since the Memphis-Shelby County Health Department initiated its culture-screening programme in 1969, efforts have expanded to include public and private hospitals and clinics, educational centres, and

Presented in part at the 28th General Assembly of the IUVDT, Malta, April, 1975

Address for reprints: Dr. Rendtorff, D.Sc., M.D., 800 Madison Avenue, Memphis, Tennessee 38163, USA

This study was conducted in part under contract HSM-21-71-523 with the Venereal Disease Control Division, Center for Disease Control, U.S. Public Health Service the consulting rooms of private physicians. Along with our studies on female complications of gonococcal infection (Rendtorff, Curran, Chandler, Wiser, and Robinson, 1974), culture screening of females was begun in the emergency room (ER) and maternity admission room (MAT) of the City of Memphis Hospital (CMH). The results emphasize the importance of the Urban Community Hospital as a source of case-finding for gonorrhoea in women.

\section{Material and methods}

The participants (except MAT) were instructed to collect cervical swabs, Z-streaking them upon Thayer-Martin (T-M) medium (Thayer and Martin, 1966), and placing them within the hour in a candle-extinction jar at $36^{\circ} \mathrm{C}$. Vaginal swabs were taken in the MAT (see below). Specimens were transported daily to the laboratory where they were further incubated and read after 24 and $48 \mathrm{hrs}$. Criteria for the identification of Neisseria gonorrhoeae were colony morphology, oxidase reaction, and Gramstain. All women with positive cultures were treated according to U.S. Public Health Service Recommendations. The sources of patients presented in this study are grouped by major categories which agree as closely as possible with those reported by the Venereal Disease Control Division, of the Centre for Disease Control Morbidity and Mortality Weekly Reports (1974). The City of Memphis Hospital sources include the ER, MAT, and Obstetric-Gynaecology outpatient clinic (OB/GYN). The patients seen in the OB/GYN Clinic were women with obstetrical or gynecological complaints as well as those attending for prenatal examinations.

Most patients from whom specimens were collected in the ER were women suspected of having pelvic infection. The maternity service of the CMH delivered approximately 7,000 babies each year of this study. Patients presenting to the MAT were in labour or had complaints in late pregnancy. A house staff physician or medical student performed a nitrazine paper test for ruptured membranes routinely on patients presenting in labour. Since it was the policy not to carry out speculum examinations on patients in labour unless specifically indicated, a vaginal swab was taken from these women. Women in active labour, with ruptured membranes, or other conditions requiring immediate attention were admitted. Others were sent home after a period of observation. 


\section{Results}

The Table presents gonorrhoea culture screening data comparing various sources of tests done in Shelby County from January, 1973, to December, 1974. While these results represent cultures, the figures closely approximate the number of individual women cultured.

As noted in the Table, 5,806 positive culture results were reported in the 2-year period. For comparison, of 18,242 women cultured in the Health Department Venereal Disease Clinic during this time, 7,070 (38.8 per cent.) were positive. The detection and treatment of an additional 5,806 females with gonorrhoea indentified by the screening programme represents an additional 82 per cent. of positive cases.

The last two columns in the Table relate the percentage of total tests done and total positive cultures detected from each source. While providing only one-third of the samples, $\mathrm{CMH}$ accounted for over one-half of the positive cultures. This is due to the high rate of positive cultures from patients in the ER.

Among test sources outside $\mathrm{CMH}$, the Health Department Family Planning Clinics accounted for the majority of both cultures and positive results detected. The $\mathrm{CMH}$ and Health Department Family Planning Clinics together accounted for 90 per cent. of the positive cultures detected in the screening programme.

\section{Discussion}

The data presented clearly show that the $\mathrm{CMH}$, an Urban Community Hospital, is a very important source for gonorrhoea case-finding in women.*

* The patients presenting to the hospital and to the Health Department Family Planning Clinics and the Community Health Centres were drawn primarily from the medically indigent who attend public health care institutions in the Memphis metropolitan area with its population of about 750,000 .
Before we started our studies, very few cultures for gonorrhoea were taken from females in the CMH-ER. Since 1972, over 800 cases a year have been diagnosed and reported from that department. Intensive sampling has indicated that over 1,900 female patients with gonorrhoea present for gyneacological assessment to the CMH-ER in a year (Curran, Rendtorff, Chandler, Wiser, and Robinson, 1975). Thus, even this productive surveillance effort is underutilized. Since most of these patients present with genitourinary or pelvic symptoms, many would be treated anyway. The culture screening systems serves as a reminder to the clinician that adequate treatment for gonorrhoea should be included in his regime for patients with pelvic inflammatory disease.

In addition, the ER surveillance system has served to point out to Venereal Disease Clinic personnel the toll extracted by gonococcal infections in terms of human morbidity and economic cost (Rendtorff and others, 1974). Also, the Health Department follow-up provides a mechanism to assure adequate test of cure as well as treatment of sex contacts. Male sex contacts are of crucial importance in this respect, since Handsfield, Lipman, Harnisch, Tronca, and Holmes (1974) observed that 57 per cent. of infected males in contact with women with symptomatic gonorrhoea are themselves asymptomatic. Reinfection from such male contacts is a common cause of recurrent pelvic infection in women.

The rate of 4.9 per cent. of positive results in the $O B /$ GYN Clinic is comparable to other studies conducted in clinics attended by pregnant patients (Waters and Roulston, 1969; Charles, Cohen, Kass, Richman, 1970). A rate of 3.5 per cent. among patients presenting in labour has not been previously reported, however. Since vaginal cultures are only 80 per cent. as sensitive as cervical cultures in the detection of gonorrhoea (Rendtorff, Chandler, Curran, 1973; Schmale. Martin, Domescik, 1969), the true infection

TABLE Results of gonorrhoea culture tests on females. Memphis-Shelby County/fanuary, 1973, to December, 1974

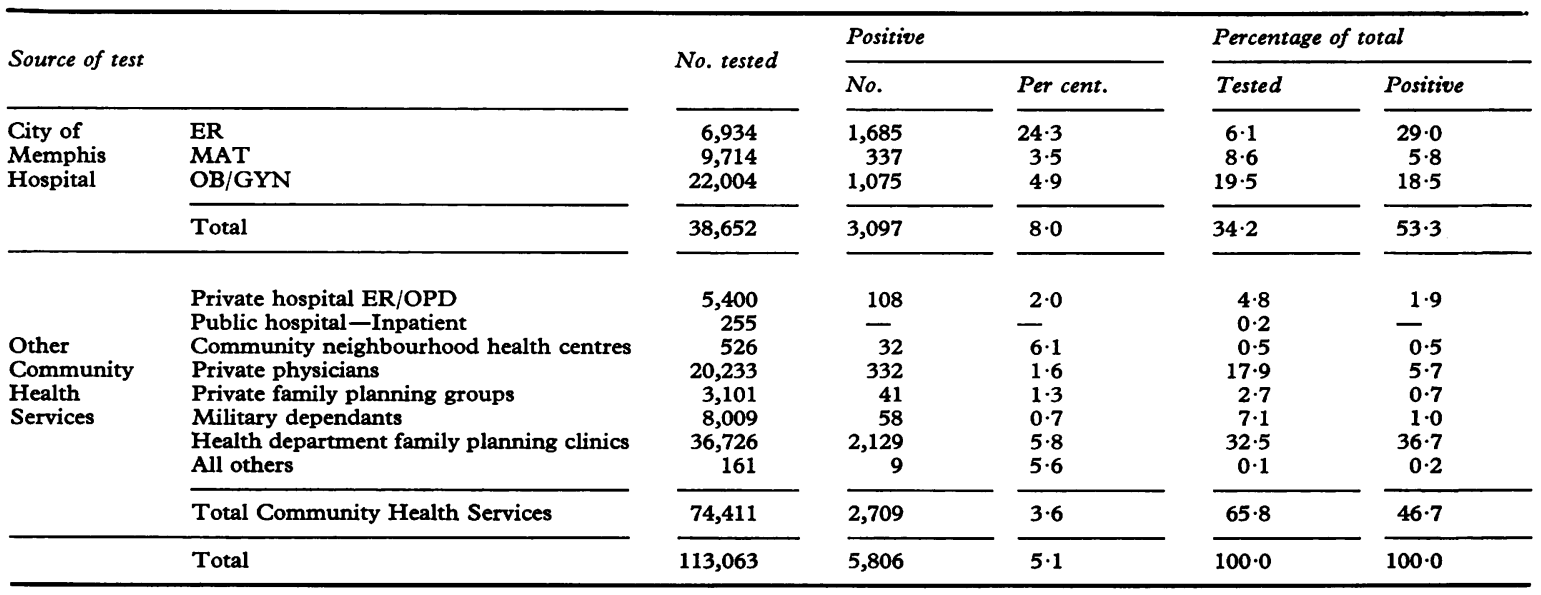


rate among these patients is closer to 4.4 per cent. Since 65 per cent. of these patients had been previously seen (and cultured) in the OB/GYN Clinic and an additional 17 per cent. had attended other sources of samples for prenatal care, many of which were participating in the screening programme, the persistently high positivity rate in these patients implies a high rate of re-infection, possibly from asymptomatic male carriers. Neonatal orogastric contamination has been correlated with prematurity, histological evidence of chorioamnionitis, and a clinical diagnosis of neonatal sepsis by Handsfield, Hodson and Holmes (1973). Futhermore, Thompson, Swanson, and Wiesner (1974), reporting on gonococcal ophthalmia neonatorum, suggested that this disorder may occur frequently despite silver nitrate prophylaxis. Because of the possibility of post-natal transmission, the detection and treatment of maternal infection is sometimes necessary to control ophthalmia neonatorum.

The above data stress the importance of including the urban community hospital in surveillance studies of gonorrhoea in women. The high rate of positive results among females seen in the emergency room identifies this area as an important reporting source. The examination and treatment of the male sex contacts of these patients may be especially helpful in preventing recurrent pelvic symptoms in them, as well as identifying potentially high-risk disseminators of infection.
The persistently high rate of positive results among parturient patients demonstrates the need to trace the male sex contacts of these patients as well. In addition, late third trimester and delivery room screening for gonorrhoea may be necessary to prevent late complications of pregnancy and neonatal gonococcal infection.

\section{References}

Center for Disease Control (1974) Morbidity and Mortality Weekly Report, 23, 231

Charles, A. G., Cohen, S., Kass, M. B., and Richman, R. (1970) Amer. F. Obstet. Gynec., 108, 595

Curran, J. W., RendtorfF, R. C. Chandler, R. W., WISER, W. L., and RoBinson, H. (1975) Obstet. and Gynec., 45, 195

HANDSFIELD, H. H., Hodson, W. A., and Holmes, K. K. (1973) f. Amer. med. Ass., 225, 697

- Lipman, T. O., Harnisch, J. P., Tronca, E., and HolmEs, K. K. (1974) New Engl.f. Med., 290, 117

Rendtorff, R. C., Chandler, R. W., and Curran, J. W. (1973) Unpublished

-, Curran, J. W., Chandler, R., Wiser, W. L., and Robinson, H. (1974) f. Amer. vener. Dis. Ass., 1, 40

Schmale, J. D., Martin, J. E., and Domescik, G. (196 9 F. Amer. med. Ass., 210, 312

Thayer, J. D., and Martin, J. E., Jr. (1966) Publ. Hlth Rep. (Wash.), 81, 559

Thompson, T. R., Swanson, R. E., and WIEsneR, P. J. (1974) f. Amer. med. Ass., 228, 186

Waters, J. R., and Roulston, T. M. (1969) Amer. F. Obstet. Gynec., 103, 532 